\title{
On the design and prototype manufacturing of multi-speed transmission device of bicycle
}

\author{
Tzu-Hsia Chen ${ }^{1}$ and Long-Chang Hsieh ${ }^{2, *}$ \\ ${ }^{1}$ Department of Mechanical Engineering, Minghsin University of Science and Technology, ROC. \\ ${ }^{2}$ Department of Power Mechanical Engineering, National Formosa University, R.O.C.
}

\begin{abstract}
The transmission systems of bicycles are multi-speed systems to satisfy different kinematic requirements. The transmission system of a bicycle can be the external changing-speed system or internal multi-speed system. A complete multi-speed transmission device of a bicycle must contain multi-speed gear unit, control device, and operating device. This paper focused on the systematic design and prototype manufacturing of multi-speed transmission device of a bicycle. Based on the results of kinematic design and meshing efficiency analysis, the systematic design of six-speed transmission device was carried out and prototype was manufactured to verify the design theorem.
\end{abstract}

\section{Introduction}

Because of the advantages of light weight, cheap, and pollution-free, bicycles become the more and more popular. They can be used as exercising machines and traffic vehicles. A complete multi-speed transmission device of a bicycle, shown in figure 1, must contain multi-speed gear unit, control device, and operating device.

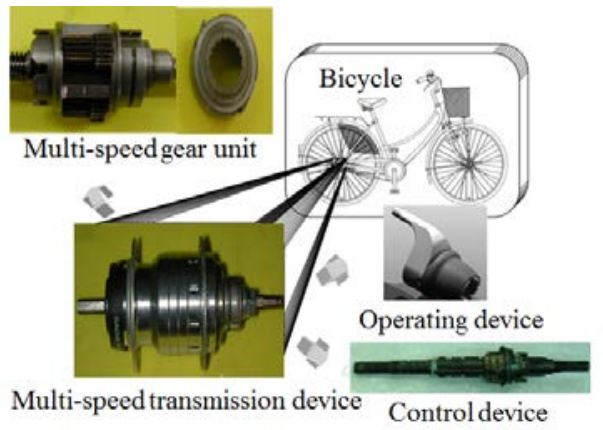

Fig. 1. Multi-speed transmission device of a bicycle.

Planetary gear trains are commonly used in various transmissions due to the reason of compact size, light weight, and multi-degrees of freedom. One of its applications is used as

*Corresponding author: lochsieh@nfu.edu.tw 
multi-speed transmission device of the bicycle. This paper focused on the systematic design and prototype manufacturing of multi-speed transmission device of a bicycle. The research subjects of planetary gear trains can be kinematic analysis, kinematic design, and meshing efficiency analysis. Traditionally, the kinematic analysis of planetary gear trains can be accomplished by tabular method or vector method. However, the procedures of such methods are complicated and tedious, often induce human errors. Fundamental circuit method [1-2] is a logical method for the analysis the angular velocities of all links of planetary gear trains. However, train value method [3-4] is the simplest one that is used for the kinematic analysis of planetary gear trains. According to the kinematic analysis, many studies focused on the kinematic design [5-8] of planetary gear trains for specified engineering purpose. The meshing efficiency of planetary gear train is an important factor of planetary gear train. There are some studies focused on the meshing efficiency analysis of planetary gear train. Based on the latent power method [9-14], the meshing efficiency of planetary gear train can be analyzed.

By referring to the train value equation of planetary gear train, the kinematic design algorithm of the multi-speed planetary gear unit was proposed. Based on the latent power theorem, the meshing efficiency equation of planetary gear unit was derived. Then, according to the meshing efficiency of ordinary gear pairs [15-17], the meshing efficiency of six-speed planetary-gear unit was analyzed. Finally, the engineering design of six-speed gear unit (including control device) was accomplished and the corresponding prototype was manufactured to verify the design theorem. In this paper, the control device and operating device were also designed.

\section{Train value equation}

For a planetary gear train, shown in figure 2, let us denote the first sun gear as $i$, the last sun gear as $j$, and the carrier (arm) as k, respectively, the train circuit can be denoted as $(i, j$; $k)$. The relationship among $\omega_{i}, \omega_{j}$, and $\omega_{k}$ can be expressed as:

$$
\omega_{i}-\xi_{j i} \omega_{j}+\left(\xi_{j i}-1\right) \omega_{k}=0
$$

Fig. 2. Planetary gear train.

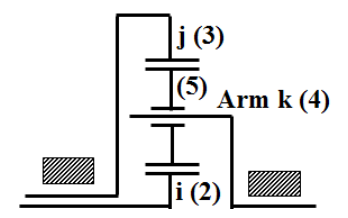

where $\xi_{j i}$ is the train value of sun gear $j$ to sun gear $i$. The sum of the coefficients of equation (1) is equal to 0 , i.e., $1-\xi_{j i}+\left(\xi_{j i}-1\right)=0$. For a planetary gear train with one train circuit, there are three members $(i, j$, and $k)$ can be adjacent to the input shaft, output shaft, and frame.

[Case I]: If carrier $k$ is adjacent to input shaft $\left(\omega_{k}=\omega_{i n}\right)$, and sun gear $j$ is adjacent to the output shaft $\left(\omega_{j}=\omega_{\text {out }}\right)$, according to equation (1), its reduction ratio $\left(R_{r}\right)$ can be written as:

$$
R_{r}=\frac{\omega_{\text {in }}}{\omega_{\text {out }}}=\frac{\omega_{k}}{\omega_{j}}=-\frac{-\xi_{j i}}{\left(\xi_{j i}-1\right)}=\frac{\xi_{j i}}{\left(\xi_{j i}-1\right)}
$$

For a planetary gear train with $\xi_{j i}<0$, the reduction ratio $1>R_{r}>0$. 
[Case II]: If sun gear $j$ is adjacent to input shaft $\left(\omega_{j}=\omega_{i n}\right)$, and carrier $k$ is adjacent to the output shaft $\left(\omega_{k}=\omega_{\text {out }}\right)$, according to equation (1), its reduction ratio $\left(R_{r}\right)$ can be written as:

$$
R_{r}=\frac{\omega_{i n}}{\omega_{\text {out }}}=\frac{\omega_{j}}{\omega_{k}}=-\frac{\left(\xi_{j i}-1\right)}{-\xi_{j i}}=\frac{\left(\xi_{j i}-1\right)}{\xi_{j i}}
$$

For a planetary gear train with $\xi_{j i}>1$, the reduction ratio $1>R_{r}>0$.

\section{Existing design}

There are many patents [18-20] of multi-speed gear hubs for bicycles. Figure 3 shows a 5-speed planetary-gear unit which is designed by Bergles in 1987 [18]. Table 1 shows its corresponding clutch sequence. Based on section 2, the corresponding reduction ratios of this 5-speed planetary-gear unit are also shown in table 1.

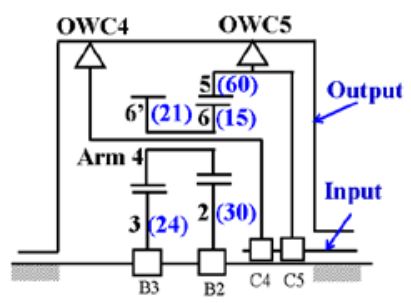

Fig. 3. Kinematic skeleton of five-speed gear unit [18].

Table 1. Clutch sequence of 5-speed planetary-gear unit.

\begin{tabular}{|c|c|c|c|c|c|c|c|c|}
\hline Speed & C4 & C5 & B2 & B3 & OWC4 & OWC5 & $R r$ & Spacing of $R r$ \\
\hline 1 & & $※$ & $※$ & & $※$ & & 1.5 & \\
\hline 2 & & $※$ & & $※$ & $※$ & & 1.2875 & 1.1651 \\
\hline 3 & & $※$ & & $※$ & & $※$ & 1 & 1.2875 \\
\hline 4 & $※$ & & & $※$ & & $※$ & 0.7778 & 1.1651 \\
\hline 5 & $※$ & & $※$ & & & $※$ & 0.6667 & 1.2875 \\
\hline
\end{tabular}

※: Engaged

\section{Kinematic design}

For a 6-speed planetary-gear unit to have the reduction ratios from 1.0 to 0.328 and the spacing of reduction ratio is constant. Table 2 shows the reduction ratio requirements of this 6-speed gear unit.

Table 2. The requirements of the 6-speed gear hub.

\begin{tabular}{|c|c|c|c|c|c|c|}
\hline Speed & 1 & 2 & 3 & 4 & 5 & 6 \\
\hline$R r$ & 1 & 0.8 & 0.64 & 0.512 & 0.410 & 0.328 \\
\hline Spacing of $R r$ & & 1.25 & 1.25 & 1.25 & 1.25 & 1.25 \\
\hline
\end{tabular}

\subsection{Design concept}

First, the design concept, shown in figure 4, is proposed for the kinematic design of 6speed planetary-gear unit. According to figure 4, the 6-speed planetary-gear unit is coupled by two planetary gear trains (I and II) in which PGT I has two independent train values $\xi_{\alpha I}$ 
and $\xi_{\alpha 2}\left(\xi_{\alpha 1}<0\right.$ and $\left.\xi_{\alpha 2}<0\right)$ and PGT II has one independent train value $\xi_{\beta}\left(\xi_{\beta>} 1\right)$. Figure 5 shows the corresponding kinematic skeleton. The reduction ratio of the planetary-gear unit is $R_{r}[\mathrm{I}] \times R_{r}[\mathrm{II}]$.

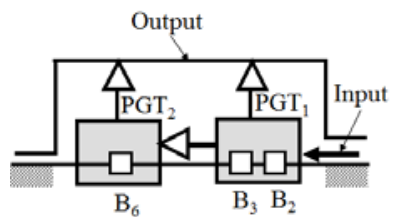

Fig. 4. Design concept.

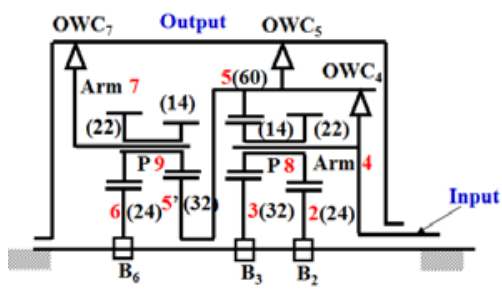

Fig. 5. Kinematic skeleton.

\subsection{One way clutch and brake}

Generally speaking, the multi-speed planetary-gear unit of bicycle includes the shifting control device (Blakes), planetary gear train, and one way clutches. One-way clutch, shown in figure 6, consists of the outer ring, rollers, inner ring, and spring (not shown). For the structure shown in figure 6(a), the rotating speed of outer ring is larger than the rotating speed of the inner ring, it is in the state of separating and represented as figure 6(c). For the structure shown in figure 6(b), the rotating speed of inner ring is larger than the rotating speed of the outer ring, it is in the state of engaging and represented as figure 6(d).

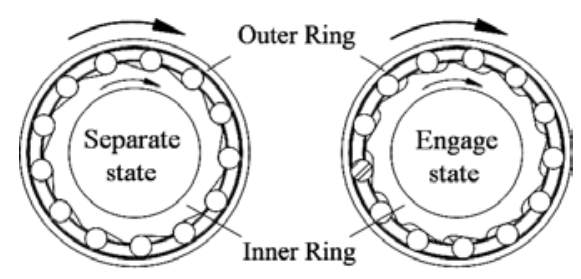

(a)Separating structure

(b) engaging structure

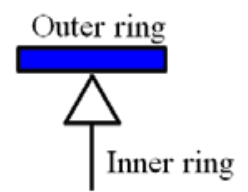

(c) separating

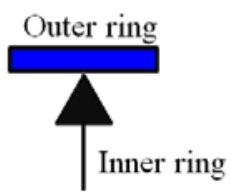

(d) engaging

Fig. 6. One-way clutch.

\subsection{Kinematic design of 6-speed planetary-gear unit}

Based on the equations. (2)-(3), the reduction ratios of the 6 speeds are expressed as:

$$
\begin{aligned}
& R_{r 1}=1.0 \\
& R_{r 2}=\xi_{\alpha 1} /\left(\xi_{\alpha 1}-1\right)=0.8 \\
& R_{r 3}=\xi_{\alpha 2} /\left(\xi_{\alpha 2}-1\right)=0.64 \\
& R_{r 4}=\left(\xi_{\beta}-1\right) / \xi_{\beta}=0.512 \\
& R_{r 5}=\left[\xi_{\alpha 1} /\left(\xi_{\alpha 1}-1\right)\right] \times\left[\left(\xi_{\beta}-1\right) / \xi_{\beta}\right]=0.41 \\
& R_{r 6}=\left[\xi_{\alpha 2} /\left(\xi_{\alpha 2}-1\right)\right] \times\left[\left(\xi_{\beta}-1\right) / \xi_{\beta}\right]=0.328
\end{aligned}
$$


According to equations. (4)-(9), if $\xi_{\alpha 1}=-4.026, \xi_{\alpha 2}=-1.914$, and $\xi_{\beta}=2.103$, the reduction ratios are $1,0.801,0.657,0.524,0.420$, and 0.344 . For the planetary-gear unit shown in figure 5, if $Z_{2}=24, Z_{3}=32, Z_{5}=60, Z_{5}=32, Z_{6}=24, Z_{8 \mathrm{a}}=22, Z_{8 b}=14, Z_{9 a}=22$, and $Z_{9 b}=22$, then the train values $\xi_{\alpha 1}=\xi_{52}=-3.9286, \quad \xi_{\alpha 2}=\xi_{53}=-1.875$, and $\xi_{\beta}=\xi_{56}=2.0952$. And, the corresponding reduction ratios are $1.0,0.797,0.652,0.523,0.417$, and 0.341 . The clutch sequence, reduction ratios, and spacing of reduction ratios are shown in table 3 .

Table 3. Clutch sequence, reduction ratios, and meshing efficiency of 6-speed planetary gear unit.

\begin{tabular}{|c|c|c|c|c|c|c|c|c|c|}
\hline Speed & B2 & B3 & B6 & OWC4 & OWC5 & OWC7 & $R r$ & Spacing of $R r$ & Meshing Efficiency \\
\hline 1 & & & & $※$ & $※$ & & 1.0 & & $100 \%$ \\
\hline 2 & $※$ & & & & $※$ & & 0.797 & 1.255 & $99.84 \%$ \\
\hline 3 & & $※$ & & & $※$ & & 0.652 & 1.222 & $99.72 \%$ \\
\hline 4 & & & $※$ & $※$ & & $※$ & 0.523 & 1.248 & $98.89 \%$ \\
\hline 5 & $※$ & & $※$ & & & $※$ & 0.417 & 1.255 & $98.73 \%$ \\
\hline 6 & & $※$ & $※$ & & & $※$ & 0.341 & 1.222 & $98.61 \%$ \\
\hline
\end{tabular}

※: Engaged

\section{Latent power theorem and meshing efficiency}

For a planetary gear train with sun gear $i$, ring gear $j$, and carrier $k$, let $T_{i}, T_{j}$, and $T_{k}$ be the torques of members $i, j$, and $k$, respectively. Since, sun gear $i$, ring gear $j$, and carrier $k$ rotate about the same axis, the torque balance equation can be expressed as:

$$
T_{i}+T_{j}+T_{k}=0
$$

Furthermore, the latent powers of sun gear $i$ and sun gear $j$ relative to carrier $k$ ( $P_{i}^{k}$ and $\left.P_{j}^{k}\right)$ are defined as:

$$
\begin{aligned}
& P_{i}^{k}=T_{i} \times\left(\omega_{i}-\omega_{k}\right) \\
& P_{j}^{k}=T_{j} \times\left(\omega_{j}-\omega_{k}\right)
\end{aligned}
$$

Let $\eta_{i j}^{k}\left(\eta_{j i}^{k}\right)$ be the meshing efficiency of planetary gear train if the latent power is transmitted from sun gear $i$ (sun gear $j$ ) to sun gear $j$ (sun gear $i$ ) when carrier $k$ is relatively fixed. Based on the concept of latent power [9-14], the relationship among $P_{i}^{k}$ and $P_{j}^{k}$ can be expressed as:

$$
\begin{aligned}
& -P_{j}^{k}=P_{i}^{k} \times \eta_{i j}^{k} \text { for } P_{i}^{k} \geq 0 \text { and } P_{j}^{k} \leq 0 \\
& -P_{i}^{k}=P_{j}^{k} \times \eta_{j i}^{k} \text { for } P_{i}^{k} \leq 0 \text { and } P_{j}^{k} \geq 0
\end{aligned}
$$

The meshing efficiency of a planetary gear train is defined as the ratio of output power to input power, i.e.,

$$
\eta_{m(P G T)}=-\frac{P_{o u t}}{P_{\text {in }}}
$$


According to equations. (10)-(14), the meshing efficiencies of case I and case II for a planetary gear train can be expressed as:

$$
\begin{aligned}
& \eta_{m(P G T)}=\frac{\eta_{i j}^{k}\left(1-\xi_{j i}\right)}{\left(1-\xi_{j i} \eta_{i j}^{k}\right)} \text { for } \xi_{j i}<0, \omega_{k}=\omega_{i n}, \omega_{i}=0, \text { and } \omega_{j}=\omega_{o u t} \\
& \eta_{m(P G T)}=\frac{\left(\xi_{j i} \eta_{i j}^{k}-1\right)}{\eta_{i j}^{k}\left(\xi_{j i}-1\right)} \text { for } \xi_{j i}>1, \omega_{j}=\omega_{i n}, \omega_{i}=0, \text { and } \omega_{k}=\omega_{o u t}
\end{aligned}
$$

where $\eta_{i j}^{k}\left(\eta_{j i}^{k}\right)$ is the meshing efficiency of planetary gear train if the latent power is transmitted from sun gear $i$ (sun gear $j$ ) to sun gear $j$ (sun gear $i$ ) when carrier $k$ is relatively fixed. The meshing efficiency of ordinary external gear pair $\left(\eta_{m(e x)}\right)$ and internal gear pair $\left(\eta_{m(i n)}\right)$ can be expressed as [15-17]:

$$
\begin{aligned}
& \eta_{m(e x)}=1-\frac{1+1 / R_{r}}{\beta_{A 1}+\beta_{R 1}}\left[\frac{f_{a}}{2} \beta_{A 1}^{2}+\frac{f_{r}}{2} \beta_{R 1}^{2}\right] \\
& \eta_{m(i n)}=1-\frac{1-1 / R_{r}}{\beta_{A 1}+\beta_{R 1}}\left[\frac{f_{a}}{2} \beta_{A 1}^{2}+\frac{f_{r}}{2} \beta_{R 1}^{2}\right]
\end{aligned}
$$

where $R r$ is the reduction ratio of gear pair, $\beta_{A 1}$ and $\beta_{A 2}$ are the angles of approach of driving and driven gears, $f_{a}$ and $f_{r}$ are the average friction coefficient of approach and recess. For external (internal) gear pair, the meshing efficiencies are between $99.2 \% \sim 99.6 \%$ $(99.7 \% \sim 99.9 \%)$. For the 6-speed planetary-gear unit, shown in figure 5 , if the meshing efficiencies of external and internal gear pairs are identified as 0.994 and 0.998 , then the meshing efficiency $\eta_{i j}^{k}=0.994 \times 0.998=0.992$ for $\xi_{j i}<0$ and $\eta_{i j}^{k}=0.994 \times 0.994=0.988$ for $\xi_{j i}>1$. Then, according to equations. (15)-(16), the meshing efficiencies of the 6 speeds are $100 \%, 99.84 \%, 99.72 \%, 98.89 \%, 98.73 \%$, and $98.61 \%$ and shown in table 3 . Based on the above results, the meshing efficiencies of planetary gear devices are very good.

\section{Engineering design and prototype manufacture}

\subsection{The design of control device}

According to the planetary gear unit, shown in figure 5, and clutch sequence, shown in table 3 , the corresponding clutch engaging diagrams of the 6 speeds are shown in figures 7(a)7(f). Based on figures 7(a)-7(f), the control device can be designed and shown in figure 8 .

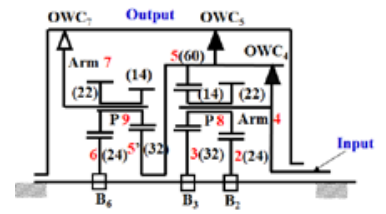

(a) $1^{\text {st }}$ gear

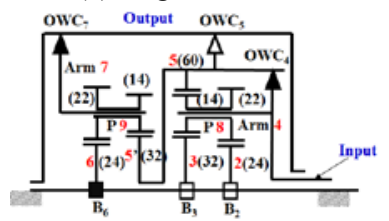

(d) $4^{\text {th }}$ gear

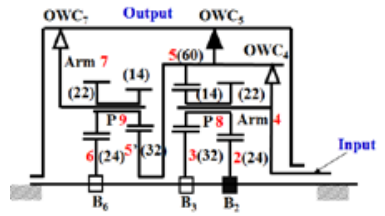

(b) $2^{\text {nd }}$ gear

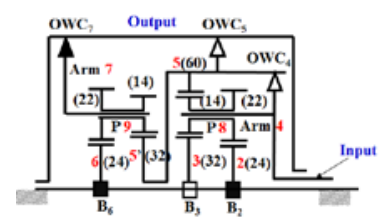

(e) $5^{\text {th }}$ gear

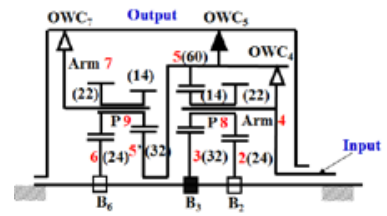

(c) $3^{\text {rd }}$ gear

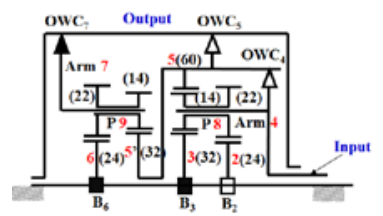

(f) $6^{\text {th }}$ gear

Fig.7. Clutch engaging diagrams of the 6 speeds. 


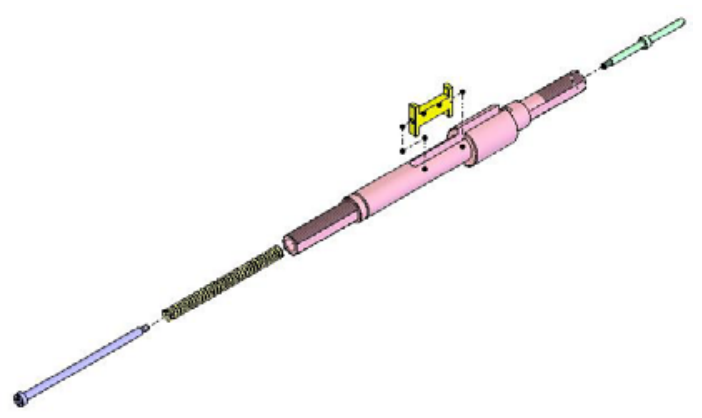

Fig. 8. Exploded view of control device.

\subsection{The design of multi-speed planetary gear unit}

According to the figure 7, the module is chosen to be 1.0, the engineering design drawings, shown in figures $9(\mathrm{a}), 9(\mathrm{~b}), 9(\mathrm{c})$, and $9(\mathrm{~d})$, are exploded view, front planetary gear train, rear planetary gear train, and one-way clutch, respectively. Figure 10 shows the dimensions of this 6-speed planetary gear hub. Figures 11(a) 11(f) show the engineering drawing of engaging diagrams of the 6 -speed planetary gear hub.

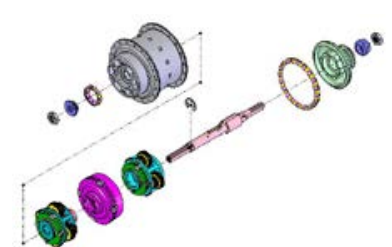

(a) Exploded view

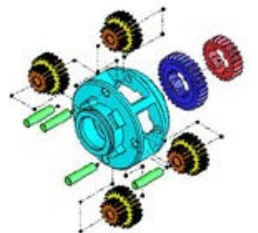

(b) Front PGT

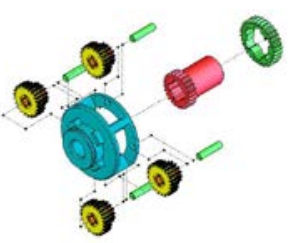

(c) Rear PGT

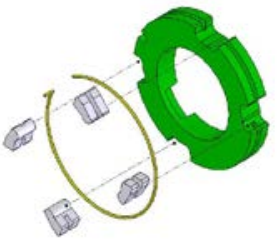

(d) One-way clutch

Fig. 9. Engineering drawings of six-speed planetary gear unit.

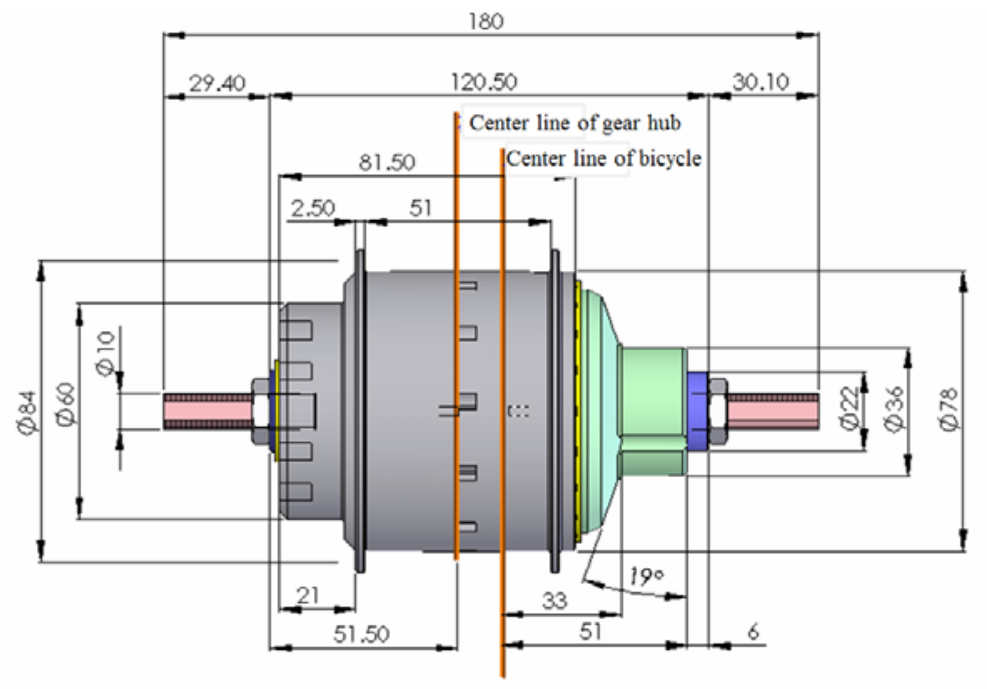

Fig. 10. Dimensions of 6-speed planetary gear unit. 


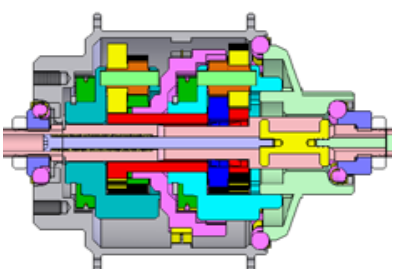

(a) $1^{\text {st }}$ speed

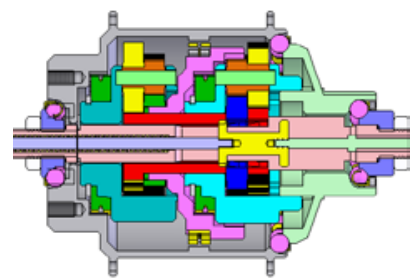

(d) $4^{\text {th }}$ speed

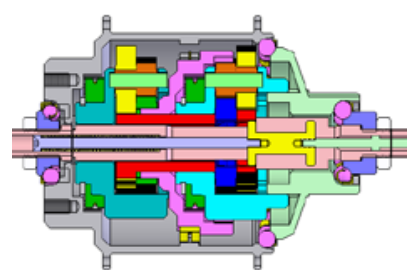

(b) $2^{\text {nd }}$ speed

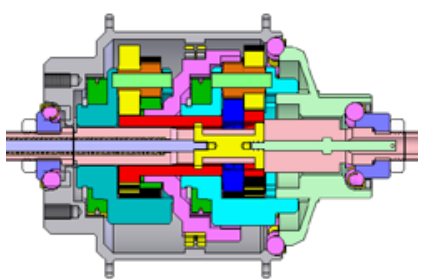

(e) $5^{\text {th }}$ speed

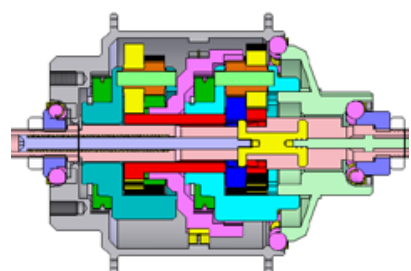

(c) $3^{\text {rd }}$ speed

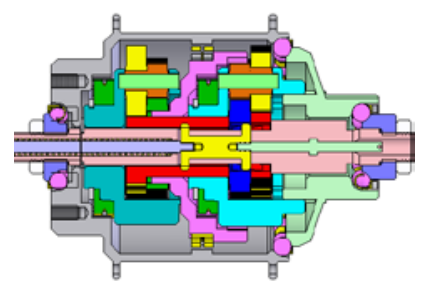

(f) $6^{\text {th }}$ speed

Fig. 11. Engineering drawing of engaging diagrams of the 6-speed planetary gear unit.

\subsection{The design of operating device}

The 6-speed transmission device includes planetary-gear unit, control device, and operating device. They are sold together. Therefore, we must design operating device at the same time. The operating device of multi-speed transmission device must contain a fixing portion, manual operating portion, positioning portion, and cable guiding portion. Also, the operating device must have the following features: simple structure, fewer parts, and low production costs. In this paper, the positioning portion and spring are designed to be one part for simplifying the structure and reducing the number of parts. Figures 13(a) and 13(b) show the outer view and the sectional view of the operating device.

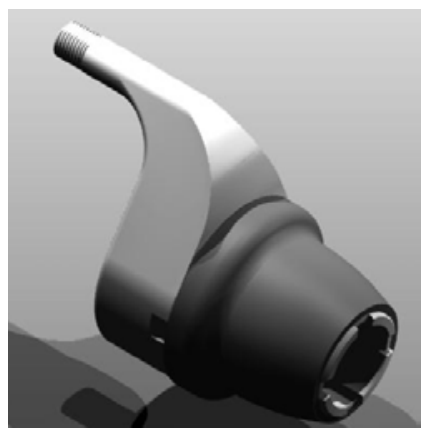

(a) Outer view

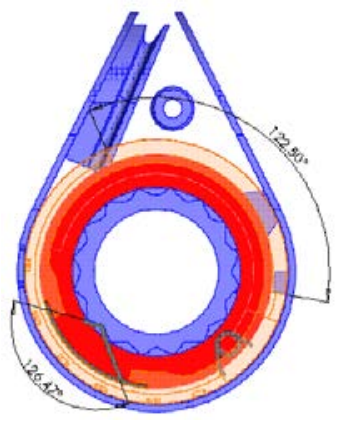

(b) Sectional view

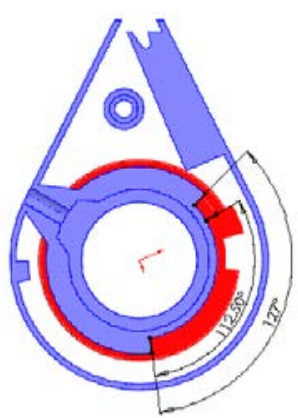

Fig.12. Operating device of 6-speed transmission device

\subsection{Prototype manufacture}

According the results of engineering design, the prototype and its parts of this 6-speed transmission device are manufactured and shown in figues 13(a)-13(f). 


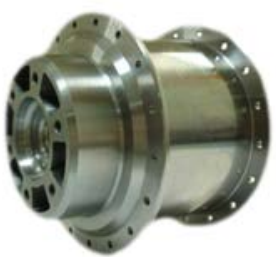

(a) Hub

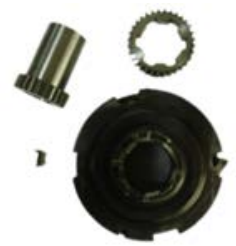

(d) Sun gears and ring gear

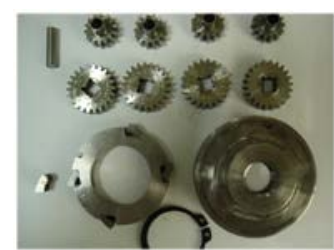

(b) Gears and carriers

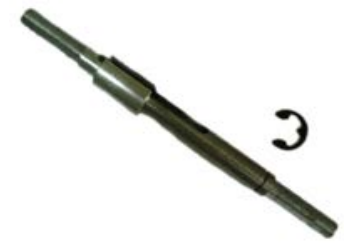

(e) Control device

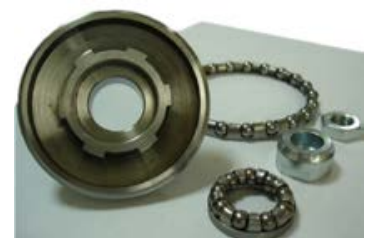

(c) Input shaft and Bearing

Fig.14. Prototype of the six-speed planetary gear unit and control device.

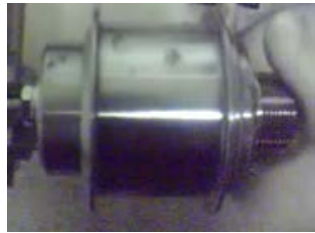

(f) Layout

\section{Conclusions}

This paper focused on the systematic design and prototype manufacturing of multi-speed transmission device of a bicycle. According to the kinematic design, a 6-speed planetary gear unit was synthesized to have reduction ratios $1,0.797,0.652,0.523,0.417,0.341$ which are good spacing. Based on the proposed design methodology, all multi-speed planetary gear units can be synthesized. According to the latent power theorem, its corresponding meshing efficiencies were calculated to be $99.84 \% \sim 98.61 \%$ for $2^{\text {nd }}$ speed $6^{\text {th }}$ speed. Finally, the engineering design of six-speed planetary gear unit was accomplished and its corresponding prototype was manufactured to verify the design theorem. In this paper, the control device and operating device were also designed.

\section{Acknowledgements}

The authors are grateful to the National Science of the Republic of China for the support of this research under MOST 104-2221-E-150-055.

\section{References}

1. L.W. Tsai, Proc. 9th Applied Mech. Conf., Kansas City, Missouri, 1, 1-5 (1985)

2. L.C. Hsieh, H.S. Yan and L.I. Wu, J. of the CSME, 10(2), 153-158 (1989)

3. C.H. Hsu, ASME J. Mech. Des, 124(3), 574-576 (2002)

4. M.C. Tsai and C.C. Huang, ASME J. Mech. Des, 132, 065001 (2010)

5. L.C. Hsieh, J.Y. Liu and M.H. Hsu, J. of TCSME, 20(4), 421-435 (1996)

6. W.M. Hwang and Y.L. Huang, J. of TCSME, 29(1), 41-55 (2005)

7. L.C. Hsieh, H.S. Lee and T.H. Chen, Materials Science Forum, 505-507, 1003-1008 (2006)

8. L.C. Hsieh and H.C. Tang, J. of AMM, 232, 955-960 (2012)

9. G. White, Mech. and Machine Theory, 38, 149-159 (2003)

10. C. Chen and J. Angeles, J. of Mechanical Design, 129, 107-113 (2007)

11. L.C. Hsieh and T.H. Chen, J. of Advanced Science Letters, 12, 34-39 (2012) 
12. L.C. Hsieh and T.H. Chen, Information-An International Interdisciplinary J. 16( 9B), 7025-7032 (2013)

13. L.C. Hsieh and H.C. Tang, J. of AIME, Article ID 686187 (2013)

14. Y.C. Wu, L.A. Chen and C.W, Chang, J. of Mechanical Engineering, 2(8), 266-273 (2014)

15. T.H. Chen, J. of TCSME, 39(3), 467-477 (2015)

16. L.C. Hsieh, T.H. Chen and H.C. Tang, J. of TCSME, 39(3), pp.455-465 (2015)

17. L.C. Hsieh and T.H. Chen, J. of TCSME, 40(5), pp.981-993 (2016)

18. E. Bergles, U.S. Patent, No.4651853 (1987)

19. G. Nurberger, U.S. Patent, No. 5443279 (1995)

20. T. Hino, US Patent No.7,967,718 (2011) 